\title{
Xenotransplantation and a Potential Threat of Animal Pathogens in Humans
}

\author{
By Liisa Sihvonen
}

National Veterinary and Food Research Institute, Department of Virology, P.O.Box 45, FIN-00581 Helsinki, Finland. Tel.: +3589 3931856, Fax: +35893931711, E-mail: liisa.sihvonen@eela.fi

\begin{abstract}
Introduction
Xenotransplantation - the transplantation of animal cells, tissues or organs into humans to treat diseases, could be an alternative to other methods. Xenotransplantation products could function for prolonged periods when given to recipients who survive for many years. Xenotransplantation is a public health concern because it has the potential to infect human recipients with infectious agents that do not ordinarily infect humans, thereby introducing new infection to humans. It combines a potential benefit with a potential risk to humans that is presently unknown.
\end{abstract}

\section{Quality control}

The quality of xenotransplantation can not be guaranteed only by control of the finished product. Great emphasis should be placed on the origin and testing of the source animal, on the controls of animal facilities and animal husbandry, on the manufacturing and on the final release testing of the product. The risk of infectious disease is mainly dependent on the choice of source animal to be used. Each species will raise its own microbiological and virological concerns which should be addressed.

Six groups of viral infections that theoretically may be transmitted to humans are listed as examples. Each group of viral infection has its own risk level.

\footnotetext{
1. Zoonoses, such as influenza A, Nipah, rabies and others,
}

2. Possible or potential zoonoses, such as porcine endogenous retroviruses (PERVs), and others,

3. Common animal viruses that normally do not infect man, such as classical swine fever, cytomegalovirus, parvovirus, and others,

4. Viruses that normally do not infect pigs (or any animal species), but have incidentally been reported to infect pigs (or any animal species), which include lyphocytic choriomeningitis, Hantaan virus, and others,

5. New viruses that may arise due to recombination of retroviral human and e.g. porcine sequences, or due to the formation of pseudotypes, e.g. a hybrid virus carrying the genome of one virus and (part of) the envelope of another, or re-assortment in the case of influenza viruses.

6. Yet unknown viruses. For example in the last years DNA sequences of three novel different gammaherpesviruses have been reported, indicating there is a chance for the presence of yet unknown pig viruses in pigs.

\section{Source of animals and testing for infectious agents}

A wide range of source animals is possible. The strategy adapted should be such as minimize potential microbiological and virological contamination. Founder animals are the animals from which source animals are initially bred. Source animals to the animal barrier facility could be derived using three different systems: 
1. Germ-free gnotobiotic founder animals. The establishment of gnotobiotic animals requires delivery by hysterectomy and maintenance in isolators under positive air pressure for the entire life span. These animals are devoid of all infectious agents except for those that are transmitted in the germline, e.g. endogenous retrovirus, or via intrauterine or transplacental pathways. 2. Specific pathogen free (SPF) founder animals. The establishment of SPF animals can be achieved by hysterectomy of the dams and maintaining SPF breeding units of the descendent animals under barrier conditions to produce source animals. 3. Founder animals free of designated pathogens. Source animals should, at minimum, be free of specific pathogens (SPF) and raised in specific facilities with barrier controls and documented health screening programs. All infectious agents known to infect the species have to be considered.

Source animals should be maintained in accordance with standard operating procedures appropriate to the species. Procedures should be developed to identify incidents that negatively affect the health of the herd or colony. Source animals should be maintained in barrier facilities. Protocols for monitoring the herd for disease and infectious agents have to exist. Specific screening procedures should include appropriate physical examination and laboratory tests. All infectious agents known to infect the source species have to be considered e.g. viruses, bacteria, mycoplasma, fungi, TSEs and parasites. All animals entering the facility have to have a defined quarantine period allowing for completion of screening procedures. The control of genetically modified animals should be at least the same as for non-genetically modified animals.

Source animal facilities have to be adequately designed and inspected by competent authorities. There have to be plans for shelter, the feed- ing area, washing areas, air handling systems and other physical attributes of the animal environment. Records of any biological or physical compromise of the animal environment and measures taken in response to this problem have to be available. When source animals die or are euthanized, a full necropsy should be performed and archival samples should be obtained for storage. When the source animal is killed at the time its live cells or tissues are harvested, a full necropsy is conducted including gross, histopathological and microbiological evaluation. Archival samples, including portions of the product, should be obtained for storage.

\section{Risk of infectious disease}

The risks of most of the viruses and the non-viral micro-organisms can be substantially reduced or even eliminated by breeding animals in a barriered environment, where they are regularly screened for the absence of infection from a wide spectrum of viruses, bacteria, parasites et cetera. It is obvious that even breeding and keeping animals under barriered conditions may not exclude hitherto unknown viruses. It is also difficult, if not impossible, to safeguard against novel viruses that emerge for recombination events. A major concern is the potential for the occurrence and spread of unknown, as well as known, animal diseases into man. Methods to remove PERVs from the genome, are not currently available, and these viruses pose the most obvious risk at present.

\section{Conclusions}

Xenotransplantation carries a significant risk of infection, not only for the recipient but also for the population at large. It is possible that in an immunosuppressed host a xenogeneic infection may occur. Transmission of pathogens from transplanted animal cells to the human host may be involved, or new diseases may arise in 
the host caused by hitherto unknown pathogens. A disease may be established and transmitted to others in the community. There might also be difficulties in recognising and diagnosing such an event at an early stage of the evolvement of such new diseases.

\section{References}

1. European Agency for the Evaluation of Medicinal Products, CPMP, Points to consider on xenogeneic cell therapy medicinal products, CPMP/ 1199/02 draft (www.eudra.org/emea.html)
2. European Commission, Scientific Committee on Medicinal Products and Medicinal Devices. Opinion on the state of the art concerning xenotransplantation. 1.10.2001

3. FDA, PHS Guideline on infectious disease issues in xenotransplantation 19.1.2001 (www.fda.gov)

4. Gunzburg WH, Salmons B. Xenotransplantation: is the risk of viral infection as great as we thought? Mol. Med. Today 2000, 6, 199-208.

5. Onions DE, Witt C. Xenotransplantation: an overview of microbiological risks and potentials for risk management. Rev. Sci. Tech. Off. Int. Epizoot. 2000, 19, 289-301. 